\title{
La Clé des Champs
}

Robbes Didier, Corentin Jorel didier.robbes@unicaen.fr

\section{UFR SCIENCES UCBN et GREYC - UMR 6072, 6 Bd Mal Juin, 14050 CAEN Cedex}

\begin{abstract}
RESUME : La problématique de la visualisation des champs électriques et magnétiques qui nous entourent est abordée sous l'angle d'expériences destinées au grand public. La démarche de cette présentation est analysée dans le détail, car elle s'appuie sur le ressort inhabituel de la poésie, pour susciter curiosité et interrogations. Au delà de cette opération ty pique de celles attendues lors de manifestations comme « la fête de la science », une transformation de la maquette initiale et de sa présentation formelle est proposée. Intégrable dans le cursus de formations de niveau L en physique et sciences de l'ingénieur, elle complète des manipulations de cartographie de champ électrique et magnétique jusqu'à l'obtention de cartographie du vecteur densité de puissance, ou vecteur de Poynting. Ainsi, la problématique de la détermination de la puissance transmise par une ligne sans contact, par échantillonnage spatial des champs électrique et magnétique autour des conducteurs constituant la ligne est abordée, au travers de mesures autour de dispositifs d'éclairage halogène à basse tension.
\end{abstract}

Mots clés : Champ électrique, champ magnétique, vecteur de Poynting.

\section{INTRODUCTION}

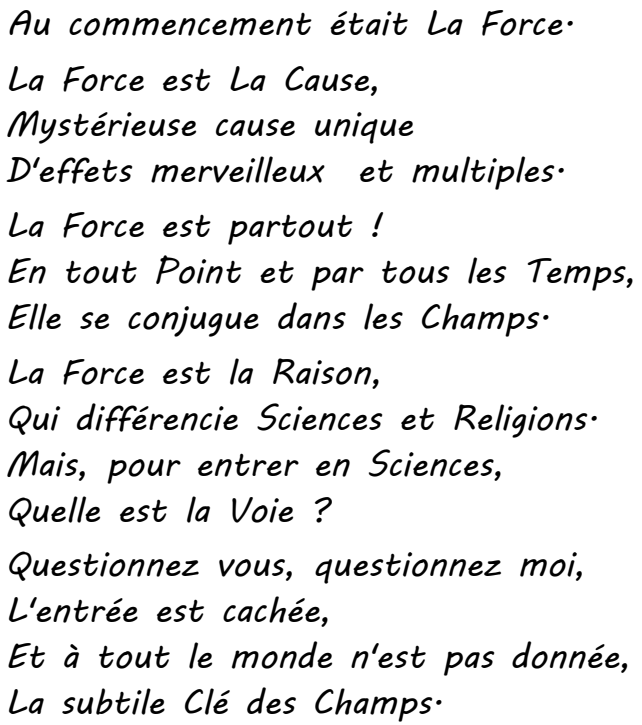

Tel était le poème que nous invitions à lire aux passants de la fête de la science, édition 2012 organisée à Caen, ce début d'automne (voir Figure -1- et -2-). Ce point d'entrée plutôt inhabituel dans nos disciplines est néanmoins un aiguillon très efficace de la curiosité des petits et grands, lors de ces manifestations ouvertes au grand public pour démystifier la science, et, au delà, inviter à la réflexion scientifique. Ici, le commentaire de ce texte poétique n'est pas seulement de nature littéraire, des expériences simples en illustrent les versets :

- illustration du concept de force : gravité, force électromagnétique, soit 2 des interactions fondamentales parmi 4 (interaction faible et forte non introduites ici.)

- visualisation du champ magnétique (par capteur présenté comme « œil de champ »),

- visualisation de ce même champ autour des fils alimentant une ampoule électrique,
- démonstration de l'existence d'un autre champ autour du même fil (mesuré par un autre œil de champ),

- $\quad$ sensibilisation à la démonstration argumentée que les deux « yeux » ou «capteurs » ne mesurent pas la même grandeur mais que ces grandeurs sont toutes les deux liées au fait que la lampe est allumée ou non

- discussion de la présence de ces champs dans nos cadres de vie habituels.

Au delà de cette série d'expériences qualitatives et de raisonnements élémentaires, une mise en forme est proposée dans le développement de cet article. Sa mise en place est prévue pour le semestre 4 dans le cadre de travaux pratiques d'électromagnétisme de la nouvelle licence SPI de l'université de Caen Basse Normandie. Cette contribution est organisée de la façon suivante. La partie 2 développe la démarche présentée lors de la fête de la science. La partie 3 donne la projection et les résultats préliminaires du matériel à finaliser pour le second semestre de cette année universitaire en cours.

\section{LA CLÉ DES CHAMPS}

\subsection{Préliminaires et fondements de la démarche}

Cette présentation grand public est dans la lignée d'actions de long terme, plus ou moins heureuses, engagées depuis une dizaine d'années autour de l'approche pédagogique par voie expérimentale des fondamentaux d'électromagnétisme et d'électrocinétique. En particulier, nous nous intéressons plus particulièrement aux difficultés multiples que présente l'appropriation intellectuelle de l'algèbre vectorielle et de ses nombreuses mises en œuvre nécessaires dans les domaines de la physique et des sciences pour l'ingénieur [1, 2, 3, 4]. 


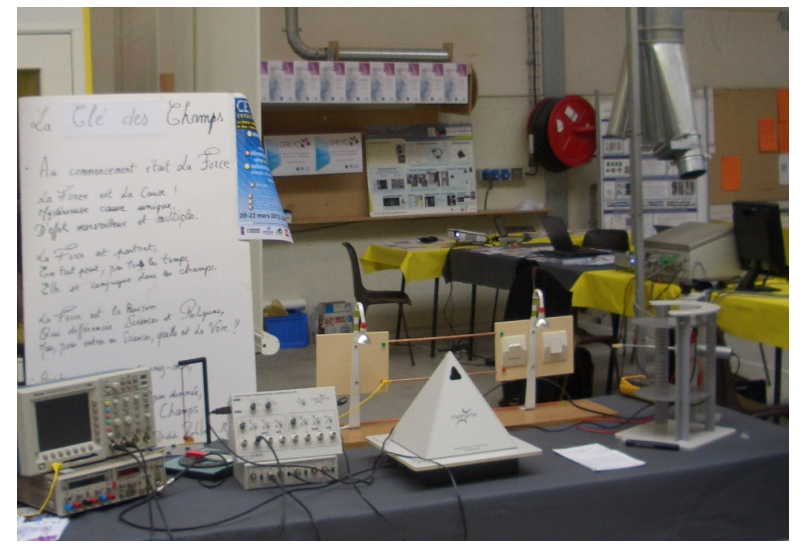

fig1 : Le stand " la clé des champs », fête de la science, Norlanda, Caen, Octobre 2012, coté stand

Les champs les plus aisés, sur lesquels il est possible de fonder des expériences probantes illustrant les opérations élémentaires d'algèbre vectorielle, sont, par excellence, les champs électriques et magnétiques, car ils s'étendent naturellement en espace libre. D'autres champs vectoriels, non moins importants, s'étendent dans les phases solides et liquides et sont donc plus difficiles d'accès par échantillonnage spatial et/ou ils sont régis par des systèmes différentiels plus complexes. Or, la nature de nos sociétés post-industrielles, grosses consommatrices d'énergie convoyée par voies électrique, d'une part, et plus encore consommatrices d'informations convoyée par voie électromagnétique, d'autre part, rend très présents à l'esprit du grand public de nombreux questionnements. Citons ceux relatifs aux effets du rayonnement électromagnétique sur la santé, et ceux relatifs aux choix de transformation d'énergie, entre les solutions hyper centralisées, d'origine nucléaire notamment, et les solutions distribuées (solaire, éolien, hydrolien, hydraulique, etc). En tant qu'enseignant chercheur, quels éléments de réponse pouvons nous apporter à ces questionnements légitimes ? Comment les rendre suffisamment intelligibles? y compris aux plus jeunes? Tel était notre questionnement pour choisir la thématique de notre présentation à la fête de la science. Nous y avons ajouté la contrainte de mutualisation possible avec l'activité habituelle d'adaptation de nos enseignements, ce qui a l'avantage de diminuer la dispersion de nos activités. Nous en rendons compte au travers de cette contribution.

Nos spécialités portant sur la mesure au travers de la mise en œuvre de capteurs variés, il suffisait, nous semble $-t$ - il, de rebaptiser ces derniers en « oeil qui voit l'invisible », et le rend visible, en vrai couleur pour notre véritable œil cette fois, sur un écran. Ceci constitue le départ du chemin vers la clé des champs. Décrivons maintenant la mise en place effective.

\subsection{Mise en ouvre pratique}

Les figures -1- et -2- donnent une idée du matériel déployé et du public lors de la manifestation. Fig. -1-, en arrière de la pyramide aisément identifiable, le disposi-

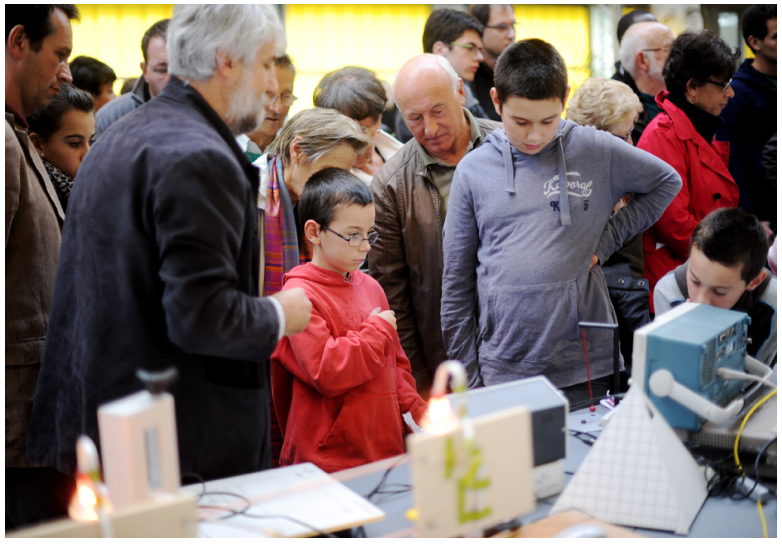

fig2 : Le stand " la clé des champs », fête de la science, Norlanda, Caen, Octobre 2012, coté public, en réflexion.

tif pour les manipulations est visible, notamment les deux ampoules halogènes à basse tension, et deux lignes de cuivre qui les relient.

\subsubsection{Matériels déployés}

Nos « yeux » pour le champ électrique et le champ magnétique ont été précédemment décrits, notamment lors de contributions aux précédents CETSIS[2, 3, 4]. Ils sont visibles sur la figure -3-. La mesure du champ électrique nous est pratiquement toujours spécifique, au moins pour le caractère directionnel de la mesure dans un volume actif suffisamment petit, facile à positionner et sans perturbation significative dans l'espace autour de la ligne bifilaire élargie convoyant l'énergie électrique aux lampes. Notons cependant des évolutions récentes d'analyseurs commerciaux de l'activité électrique et magnétique ambiante, permettant désormais l'identification, simultanée et sur 3 axes, des 3 composantes du champs magnétique et des 3 composantes du champ électrique [5]. Ces analyseurs, qui peuvent donc ressortir les composantes du flux de puissance, seraient toutefois difficiles à utiliser pour notre expérience, les capteurs y étant intégrés soit dans le boîtier de l'instrument, ou de la taille d'un gros œuf. L'ensemble peut difficilement être considéré comme quasi ponctuel, et rend caduque la notion d'échantillonnage spatial qu'il est pourtant nécessaire de mettre en œuvre dès lors que l'on souhaite mettre en évidence une grandeur vectorielle, définie en chaque point de l'espace.

A coté des « yeux » qui voient l'invisible, l'écran d'un oscilloscope numérique couleur permet de restituer une image bien identifiable, y compris par les plus jeunes, moyennant certaines précautions que nous détaillerons lors de la présentation du protocole de déroulement des manipulations présentées. L'oscilloscope est complété par un traditionnel générateur basse fréquence.

Pour différencier les fonctions des capteurs, deux dispositifs sources de variation de champ magnétique et de champ électriques sont mises en place, et alimentées par le générateur de signal basse fréquence. Ces sources, reportées en figure -5- et -6-, permettent d'ob- 


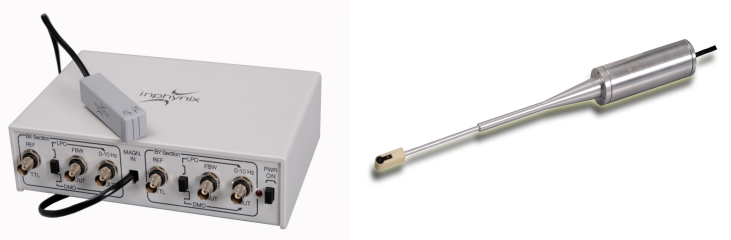

Fig 3. A gauche: capteur magnétique et conditionneur. A droite : capteur de champ électrique.

tenir, dans une région connue de l'espace, un champ électrique et un champ magnétique dominant les contributions parasites.

Pour rendre perceptible à nos sens (vue et toucher) la notion de force, car c'est le point de départ du poème et de nos expériences, deux pendules modifiés sont utilisés. Le premier, photographié figure - 4 - à gauche est composé d'un balancier rigide, mobile autour d'un point fixe, chargé par une masse aimantée. Le socle peut également recevoir des aimants, modifiant profondément la dynamique du pendule libre. Il illustre les effets conjoints de la force de gravitation et de la force magnétique. L'objet, bien évidemment de nature ludique, attire également très efficacement l'attention. Pour rendre également bien perceptible directement à nos sens les effets de l'interaction coulombienne entre deux sphères très légères et électrisables, Le second pendule a été utilisé [4]. Une photographie est donnée Fig -4-, à droite.

\subsubsection{Expériences et poésie}

Le premier vers du poème pose la notion primordiale de force : «Au commencement était La Force »» $\mathrm{Au}$ delà du triple clin d'œil à la référence biblique, au Graal de la physique (problématique de la grande unification) ou encore à la guerre des étoiles, les spectateurs étaient appelés, après lecture du poème, à réfléchir autour du pendule simple, constitué d'une simple masse et d'un fil rigide. La force de gravitation en cause dans ce système est naturellement très bien identifiée par la grande majorité des personnes, avec les notions de position d'équilibre et de verticale.

En seconde expérience, l'équilibre à la verticale est empêché par la présence d'un aimant orienté en opposition de celui constituant la masse du pendule. La force magnétique, intervenant alors en plus de la gravité, est perçue de façon visuelle par la modification de la posi-
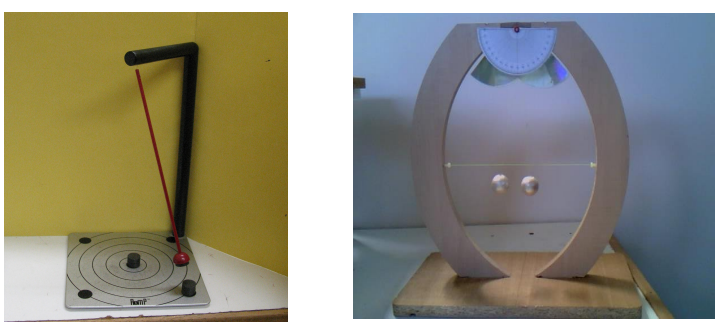

Fig 4. Image de Gauche : Pendule à masse magnétique et aimants perturbateurs. Image de droite : pendule double visualisant l'effet de la force coulombienne.

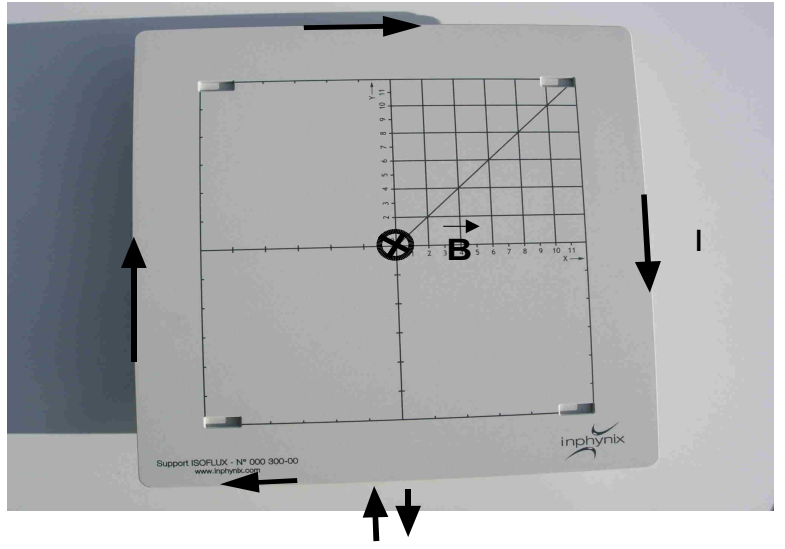

fig 5. Source de champ magnétique seul, vue de dessus. Le courant source, marqué par des flèches, circule dans un bobinage (non visible) enroulé sur gorge dans le plateau.

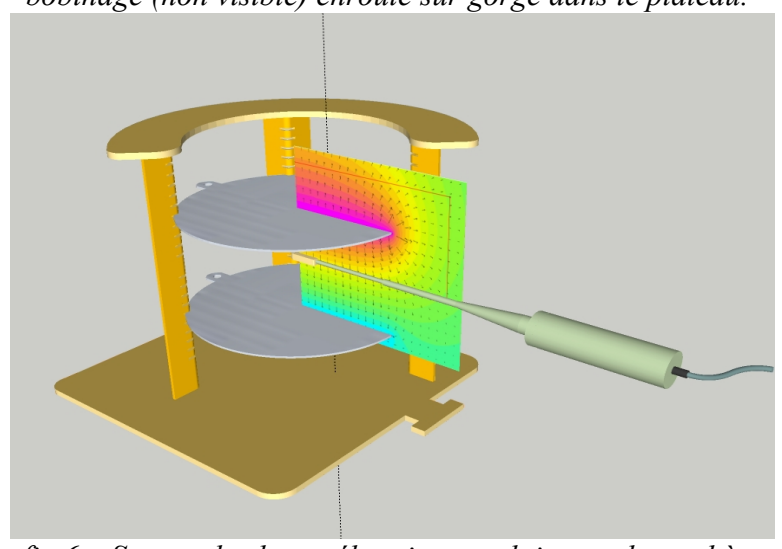

fig 6. Source de champ électrique seul, image de synthèse pour visualiser le champ électrique associé

tion d'équilibre (plus de $15^{\circ}$ ). Elle peut être également ressentie par le toucher, en pinçant entre 2 doigts, le pendule au dessus de l'aimant perturbateur. La nouvelle position d'équilibre n'est pas bien définie, en raison de la symétrie de rotation autour de la verticale. Un nouvel équilibre est obtenu avec un second aimant par effet attractif. A l'issue de ces 2 expériences, la coexistence de forces agissant sur le même objet est introduite, incluant la notion d'attraction et de répulsion. Les forces, en tant que telles, ne sont pas visibles. Elles ne le sont qu'au travers de leurs effets. Si l'on dispose plusieurs aimants au hasard sur le socle de ce premier pendule, la trajectoire de celui-ci devient erratique, d'où la strophe I du poème :

\section{La Force est La Cause, Mystérieuse cause unique D'effets merveilleux et multiples.}

Mais bien d'autre effets peuvent être obtenus, si l'on dépasse ce stade élémentaire, et que les notions d'action à distance, d'espace et de temps entrent en poème :

\section{La Force est partout! \\ En tout Point et par tous les Temps, Elle se conjugue dans les Champs.}

Figure de style ? Certes, mais les fondements de la notion de champ en physique et mathématiques s'y en- 
tendent. Pour rendre plus concrète, ou sensible, la notion abstraite de champ, le capteur magnétique est placé au voisinage du pendule à l'équilibre, puis le pendule est écarté de sa position d'équilibre, et y retourne en oscillant sur de nombreuses périodes. Simultanément, la réponse du capteur est visualisée à l'oscilloscope, dont l'écran doit être vu en même temps que le pendule en mouvement. La concordance évidente entre le déroulement de la trace et celui de la trajectoire du pendule montre donc que les deux sont fortement liés, mais ce n'est pas directement à la force magnétique que le capteur est sensible ! En effet, le déplacement manuel du capteur au voisinage de l'aimant du pendule, ne provoque pas le même effet sur lui que sur les aimants des expériences précédentes (la présence du capteur ne modifie pas la position d'équilibre, par exemple). Ainsi avons nous essayé de rendre intelligible et admissible l'existence d'un champ, baptisé magnétique, déployé tout autour des aimants, et accompagnant les mouvements dans le temps du pendule aimanté, alors que nos propres sens, seuls, ne peuvent en rendre compte.

La strophe 3 de notre poème, qui commence par : « La Force est la Raison », souligne, par son ' $R$ ' le détachement obligatoire entre nos perceptions directes des phénomènes et leurs représentations intellectuelles qu'il est possible de construire avec méthode, nous disait déjà Descartes. La strophe se poursuit par : «Qui différencie Sciences et Religions»,car, croire est le fondement même des religions - en sciences, il faut argumenter, douter, prouver, formuler des hypothèses, diagnostiquer les contradictions, etc... Les expériences présentées ciaprès illustrent alors la démarche expérimentale, composante essentielle s'il en est, de la pratique scientifique. Tel est le chemin pour "entrer en sciences", exigeant, long, ardu, mais tous les objets technologiques dans la vie de tous les jours ne sont-ils pas fondés sur les développements qui en résultent?

Enfin, l'envoi de ce poème :

\section{"Questionnez vous, questionnez moi, L'entrée est cachée, \\ Et à tout le monde n'est pas donnée, La subtile Clé des Champs",}

vise à renommer l'un des concepts abstraits essentiel et unificateur de la science moderne, le concept de champ. Il invite au questionnement et à l'échange, pour que s'affine la représentation de ce concept, via les expériences présentées et commentées ci - après.

\subsubsection{Expériences et démarche expérimentale}

La sensibilisation à la notion de champ magnétique, décrite au début du 2.1.2 étant faite, le capteur magnétique est alors placé au voisinage des lampes halogènes et de leur ligne d'alimentation. La trace correspondante de l'oscilloscope (voir Fig. -7-) indique alors la présence de champ magnétique tout autour du dispositif. La trace se réduit à un trait dès que la lampe est éteinte et cette observation est parfaitement répétable. Or, un second capteur est également placé au voisinage de la ligne, et une seconde trace de l'oscilloscope, qui lui est associée, montre un effet tout à fait semblable. Les traces correspondantes sont reportées figure -7-. La question associée à ces indications est alors : ce second capteur procure-t-il une réponse sensible au même champ que le premier capteur ?

Une expérience permet aux spectateurs de trancher: elle utilise les sources de champ reportées en Fig -5pour le champ magnétique et Fig -6- pour le champ électrique. Il est aisé de montrer que le premier capteur répond au voisinage du dispositif Fig -5-, mais ne répond pas du tout au voisinage du dispositif Fig -6-. La situation inverse est obtenue pour le second capteur. Les conclusions que l'on peut formuler à partir de ces expériences sont alors :

- les deux capteurs ne sont pas sensibles au même champ,

- ces deux champs sont présent simultanément au voisinage des fils conducteurs uniquement lorsque les lampes sont allumées,

- Les deux traces d'oscilloscope sont très semblables, indiquant que probablement ces deux champs sont fortement liés,

- Lorsque les lampes sont éteintes, la réponse magnétique disparaît, alors qu'une réponse résiduelle notable persiste pour le second capteur.

Le questionnement légitime est alors : quel est donc ce champ indiqué par ce capteur non magnétique ? La réponse à cette question peut être obtenue en rappelant aux spectateurs le phénomène d'électrisation statique d'une règle en plexiglas qui attire les petits bouts de papiers. Une telle électrisation peut se faire également sur le pendule double, reporté en Fig -4-, photographie de droite. Le résultat obtenu - les deux boules s'écartent après leur électrisation - permet ainsi de boucler sur la problématique générale abordée au début du déroulement de ces manipulations (début du 2.2.2), à partir de la notion de force. Cette fois, l'interaction newtonienne est en compétition avec l'interaction coulombienne, et la sortie du capteur de champ électrique est sensible

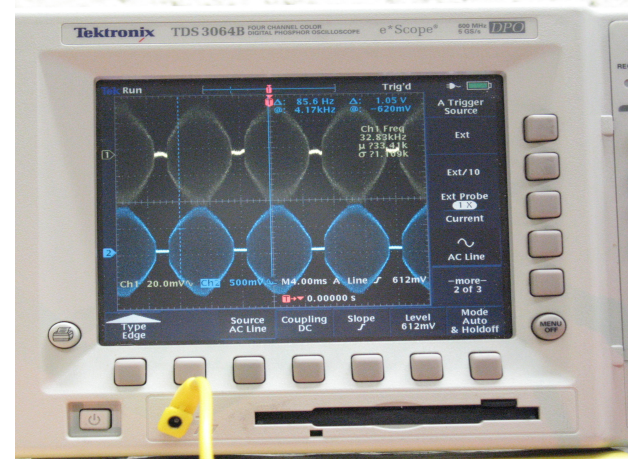

fig 7. Traces des images de champ magnétique (bleu) et de champ électrique (jaune), les capteurs étant placés entre les conducteurs de cuivre matérialisant la ligne Fig -1-. 
aux mouvements des boules de ce pendule autour de leur position d'équilibre, tout en y associant la trajectoire de la trace image sur l'écran de l'oscilloscope. La nature du second champ autour des lampes et des lignes d'alimentation est ainsi présentée, en essayant de privilégier la nature unifiée de la formalisation de la notion de champ, au travers de la notion commune de force à distance.

Cette démarche de présentation grand public nous est apparue originale et relativement efficace, compte tenu des retours que nous avons pu avoir, notamment par la fréquence et la nature des questions posées à l'issue de ces présentations. Ces retours diffèrent suivant le vécu des personnes, age, etc. Pour en faire un état correct, il aurait été nécessaire de filmer les scènes.

\section{MUTUALISATION AVEC DES TP}

Voyons maintenant les adaptations envisagées pour des travaux pratiques de niveau L2, L3, pour la physique et les sciences de l'ingénieur, mais nous pensons également que les formations de techniciens supérieurs en génie électrique notamment sont également concernés.

\subsection{Vecteur de Poynting - flux de puissance}

Les deux capteurs mis en œuvre, mesurent chacun une composante du champ, soit électrique, soit magnétique. Il suffit donc, en principe, de positionner ces capteurs l'un par rapport à l'autre pour que leurs directions respectives de sensibilité soient orthogonales. Cette contrainte étant supposée satisfaite, le produit du résultat des mesures respectives simultanées, (ou considérée par période), est donc proportionnel au flux du vecteur de Poynting au travers de l'élément de surface s'appuyant sur les directions de sensibilité de cette paire de capteurs orthogonaux. Or, avec les ergonomies actuelles des oscilloscopes numériques, le produit voie à voie est disponible, y compris en temps réel, avec les opérations arithmétiques courantes associées. Par conséquent, les affichages à l'écran d'un oscilloscope numérique 4 voies d'une composante du champ électrique, de la composante transverse du champ magnétique, et de leur produit est aisément réalisable. L'on dispose ainsi de mesure quasi ponctuelle de la densité du flux de puissance s'écoulant de la source vers la charge par l'intermédiaire de la ligne. Par conséquent, la procédure d'échantillonnage spatial introduite précédemment $[1,2,3]$ pour les champs magnétique et électriques étant parfaitement opérationnelle, elle est également applicable au vecteur densité de Poynting.

\subsection{Expérience à réaliser}

Dès que les fondements de l'électromagnétisme ont été présentés aux étudiants en physique et sciences pour l'ingénieur, deux exercices standards sont effectués en travaux dirigés, généralement sans expériences qui y soient directement rattachées. Il s'agit des calculs des champs électriques et magnétiques confinés entre deux plans parallèles limités, afin d'en ressortir la capacité par unité de longueur et l'inductance par unité de longueur de la ligne microstrip, par exemple. La conception de l'expérience que nous voulons mettre en place sur sur ce type de dispositif est maintenant abordée.

\subsubsection{Concepts de base et ordre de grandeurs}

L'idée est de réaliser une ligne « macrostrip », dont le dimensionnement soit exagéré, et dont le diélectrique soit de l'air, voir figure -8-. La taille du dispositif doit être telle que, vis à vis de la dimension de nos capteurs, ces derniers puissent retourner une série de mesures quasi ponctuelles, réalisant un maillage suffisamment fin du plan de section de la ligne. La surface sur laquelle porte cet échantillonnage devra être suffisante pour qu'au moins $90 \%$ du flux de puissance traverse l'aire échantillonnée. Cette ligne sera couplée à son entrée au générateur constitué par le convertisseur $220-$ $12 \mathrm{~V}$. En sa sortie, la lampe halogène basse tension sera connectée. Compte tenu des expériences décrites précédemment, pour ce qui était une portion de ligne bifilaire, nous savons donc que les signaux sont parfaitement mesurables, aisément comparables aux signaux de tension et de courant mesurables par contact sur les conducteurs. Ces derniers nous donnent accès au courant débité par la source, chargée par une lampe consommant la puissance de nominale de $50 \mathrm{~W}$. Le courant délivré $I_{d}$ est alors de l'ordre de $4 \mathrm{~A}$, et la charge $R_{L}$ de l'ordre de $1,7 \Omega$. L'observation montre également que la fréquence de hachage du convertisseur est de 35 $\mathrm{kHz}$. L'épaisseur de peau $\delta$ associée est alors de l'ordre de $0,37 \mathrm{~mm}$ pour le cuivre, et donc la réalisation en circuit imprimé standard, mettra en œuvre des épaisseurs conductrices bien inférieures à $\delta$. L'estimation numérique du champ magnétique peut alors se faire moyennant l'hypothèse d'une répartition superficielle de courant, de densité $\vec{j}_{s}$ donnée par :

$$
\vec{j}_{s}=\frac{I_{d}}{w} \vec{u}_{x},
$$

où $w$ est la largeur du ruban constituant la ligne, et $\vec{u}_{x}$ un vecteur unitaire dans sa direction principale. Dans ces conditions, le champ magnétique confiné entre les armatures dans la limite où leur séparation $t$ est faible devant la largeur $w$, est donné simplement par :

$$
\vec{H}=\vec{j}_{s} \wedge \vec{n}
$$

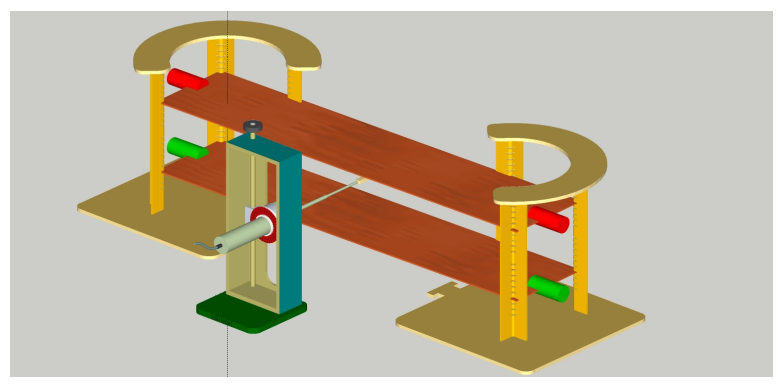

fig 8. Ligne « microstrip » agrandie à réaliser. 
$\vec{n}$ vecteur normal au plan du ruban convoyant le courant, en direction du second conducteur.

Pour $w=20 \mathrm{~cm}$, nous obtenons : $|\vec{H}| \approx 20 \mathrm{Am}^{-1}$, soit environ $20 \mu T$ en induction, une valeur parfaitement mesurable, compte tenu de l'étendue de mesure de nos capteurs et de leur sensibilité. L'analyse simplifiée de la ligne donne tout aussi directement le champ électrique confiné entre les électrodes et s'atténuant rapidement dès que l'on s'éloigne des bords :

$$
\vec{E}=\frac{V}{t} \vec{n}
$$

où $\mathrm{V}$ est la différence de potentiel entre les plans conducteurs, soit $12 \mathrm{~V}$. Pour une distance inter armature de $t=5 \mathrm{~cm}$, l'estimation du champ électrique en module est de $|\vec{E}| \approx 240 \mathrm{Vm}^{-1}$, valeur tout à fait accessible à notre électromètre. Comme le champ électrique s'atténue très rapidement en dehors des électrodes, il en est de même pour le vecteur de Poynting, justifiant après coup qu'il ne soit pas nécessaire d'estimer le champ magnétique tout autour de la structure. Au final, la densité de flux de puissance $\vec{p}$ est :

$$
\vec{p}=\vec{E} \wedge \vec{H}
$$

Partant des valeurs des champs $\vec{E}$ et $\vec{H}$ une estimation de la valeur de la densité de puissance est $|\vec{p}| \approx 4,8 \mathrm{kWm}^{-2}$. Intégrée sur la section utile $w t$, elle mène à l'estimation de la puissance transportée voisine de $48 \mathrm{~W}$. Cette valeur est bien voisine de celle attendue, puisque le convertisseur est donné pour délivrer $50 \mathrm{~W}$. Tel est ce que nous souhaitons faire vérifier aux étudiants lors de la mise en œuvre de ce TP.

\subsubsection{Déroulement prévisible du TP}

Le travail consistera à identifier les caractéristiques directionnelles des champs $\vec{E}$ et $\vec{H}$ dans la région centrale du dispositif fig -8-, afin de vérifier leur caractère transverse. Puis le travail portera sur l'identification des caractéristiques temporelles des composantes des transverses, à partir des traces mesurées à l'oscilloscope, qui seront très semblables à celles de la figure -7-. En particulier, les étudiants devront mesurer la période de l'enveloppe des signaux (20 ms), ainsi que la période du signal contenu dans cette enveloppe (28 $\mu \mathrm{s})$. Ils devront alors s'interroger sur la phase relative des deux signaux associés aux champs $\vec{E}$ et $\vec{H}$, et montrer que le produit de ces deux signaux admet une modélisation mathématique simple, si l'on décrit les signaux associés aux champs par des signaux carrés à enveloppe sinusoïdale. La valeur moyenne sur $20 \mathrm{~ms} d u$ produit de ces deux signaux devra être calculée à partir de ces mesures, pour en déduire l'estimation du vecteur de Poynting associé au point central du dispositif. Ce protocole de mesurage étant plutôt long et fastidieux, les étudiants calculeront le rapport existant entre ce résultat final et la valeur instantanée maximale obtenue directement à partir des valeurs de crête des signaux as- sociés à $\vec{E}$ et $\vec{H}$. Ce rapport devant être constant, la procédure simplifiée de mesurage de la densité de puissance transportée sera alors de prendre les valeurs crêtes des signaux images de $\vec{E}$ et $\vec{H}$, puis d'en effectuer ce produit pondéré. Dès lors, l'échantillonnage spatial de la densité de puissance au travers d'une section transverse de la ligne est une série de mesures accessible dans un temps acceptable, estimé à 6 points par minutes. Une telle cadence permet alors une cartographie de $\vec{p}$ contenant une centaine de points, résultat de son échantillonnage spatial. Son exploitation pour obtenir une estimation du flux intégré est alors également accessible par calcul au moyen d'un tableur. Le résultat doit mener à une valeur proche de la puissance mesurable classiquement, comparaison qui constituera la dernière étape de ce TP. A la charnière électromagnétisme - convoyage d'énergie, ce TP permettra à nos étudiants de L2 d'améliorer leur représentation des phénomènes mis en jeu dans ces problématiques.

\section{CONCLUSION}

Nous avons décrit un protocole associant poésie et expérience, pour contribuer à une meilleure représentation de la notion de champ par le grand public. La mise en œuvre des expériences décrites est aisée dès lors que des capteurs directionnels de champ électrique et magnétique sont quasi ponctuels. Un échantillonage spatial du vecteur de Poynting est obtenu, menant à la conception d'un nouveau TP, dont la mise en place est prévue courant 2013, dans le cadre de l'année 2 de la licence science pour l'ingénieur de l'université de Caen. Des expériences supplémentaires sont rendues possibles, notamment en basse fréquence compatibilité électromagnétique (CEM, $50 \mathrm{~Hz}$ et harmoniques).

\section{Bibliographie}

[1] D. Robbes Magnétostatique, algèbre vectorielle: une série de T.P. mise en place en DEUG STPI à Caen.. Proceedings of CETSIS'2003, Toulouse, 13-14 novembre 2003.

[2] Didier Robbes, Thierry Salley, Fréderic Dijoux, Basile Dufay, Gilles Allègre, Maria Timofeeva, S. Morteau "Autour des équations de Maxwell », actes du colloque CETSIS 2010, 8 - 10 mars 2010, Grenoble.

[3] Didier Robbes, «Physical Quantity Measuring Unit and Device for Measuring a Voltage and an Electric Field » European Patent Application No. 09290213.9 , date 20.3.2009

[4] Emmanuel Jacquet, Didier Robbes, Alain Méry, Michel Guillermic, Zahed Bahna, «Manipulations et matériels partagés entre l'enseignement secondaire et l'enseignement supérieur, une utopie? », Bulletin de l'union des physiciens, BUP, Vol 106, Fev. 2012, pp $201-215$

[5] «ESM - 100 3D field meter with FFT», www.maschek.de/pdf/ESM-100-fr.pdf 